\title{
Jóvenes en la encrucijada de la desventaja: análisis de las situaciones de desventaja social y conductas de riesgo en Vitoria-Gasteiz
}

\section{Grupo de Trabajo Interdepartamental, Ayuntamiento de Vitoria-Gasteiz}

<planjoven2@vitoria-gasteiz.org>

Gasteizko Udalaren II. Gazte Plana onartu zen 2012an, eta bertan onartzen zen arabar hiriburuan bizi diren gazteen gizarte-desabantaila eta arriskujokabideetako egoerak identifikatzeko azterlan bat burutzea. Artikulu honek laburbiltzen du aipaturiko azterlana eta gaur egun praktikan jartzen ari diren bertako eskuartzerako gomendioak.

\section{GAKO-HITZAK:}

Gizarte-desabantaila, arrisku-jokabideak, gizarteratzea, prebentzioa.
El II Plan Joven Municipal de Vitoria-Gasteiz, aprobado en 2012, contemplaba la realización de un estudio con el doble objetivo de identificar las situaciones de desventaja social y las conductas de riesgo de la juventud residente en la capital alavesa, y de proponer medidas para abordarlas. El presente artículo sintetiza dicho estudio, cuyas recomendaciones de intervención se están poniendo en práctica actualmente.

\section{Palabras Clave:}

Desventaja social, jóvenes, conductas de riesgo, inclusión social, prevención. 


\section{Presentación}

El trabajo que presenta este artículo es el resumen de un estudio elaborado por el Grupo de Trabajo Interdepartamental del Ayuntamiento de Vitoria-Gasteiz, coordinado desde el Plan Joven, que depende de la Unidad de Juventud y el Servicio de Educación municipal ${ }^{1}$. En el grupo han trabajado conjuntamente técnicos del Servicio de Centros Cívicos, del Servicio de Salud, del Departamento de Asuntos Sociales, del Servicio de Policía Local, además de Juventud y Educación ${ }^{2}$. El proceso de investigación fue apoyado por otras instituciones y asociaciones competentes en el tema, que aportaron datos al inicio del proceso y contrastaron los resultados del diagnóstico al final. Como consecuencia de este trabajo, se han diseñado acciones que el III Plan Joven Municipal (2013-2015) comienza ahora a desarrollar.

\section{Contextualización}

El II Plan Joven Municipal de Vitoria-Gasteiz (Servicio de Juventud, 2010), en su proceso de diseño, contempló la necesidad de abordar la desventaja social y las conductas de riesgo entre las personas jóvenes, que se veía como una necesidad compartida por los agentes que participaron en la elaboración de dicho Plan (asociaciones relacionadas con esta casuística, personal técnico de los departamentos con competencias en los ámbitos aquí analizados y representantes políticos de todos los grupos que en aquel momento conformaban la corporación municipal).

En primer lugar, el grupo de trabajo técnico realizó una labor de búsqueda de consenso en cuanto a qué entendíamos por situaciones de desventaja y conductas de riesgo, cómo se articulaban en la realidad de las personas jóvenes y cuáles eran las situaciones y conductas que debíamos incluir en el correspondiente análisis. Todo esto se plasmó en un documento de partida (véase el apartado 4). Después, se solicitó información a las diferentes administraciones y entidades relacionadas con el contenido de trabajo. La respuesta, en general, puede calificarse de positiva, aunque en algunos casos no se pudieran conseguir los datos de un modo segmentado, tal como se demandaban.

Recopilada la información relevante, en un trabajo bilateral entre la Unidad de Juventud y los distintos departamentos o servicios, se procedió a un diagnóstico de la situación en Vitoria-Gasteiz (véase el apartado 5). Somos conscientes de que muchos factores o situaciones de desventaja y conductas de riesgo no se pueden valorar de una manera aislada y que deben ser analizados de forma conjunta, pero sistematizar el

${ }^{1}$ Grupo de Trabajo Interdepartamental, Jóvenes en la encrucijada de la desventaja: análisis de las situaciones de desventaja social y conductas de riesgo en Vitoria-Gasteiz, Vitoria-Gasteiz, Ayuntamiento de Vitoria-Gasteiz, 2012 [/http://www.vitoria-gasteiz.org/wbo21/ http/contenidosEstaticos/adjuntos/es/88/10/48810.pdf)].

${ }^{2}$ A todos ellos, el agradecimiento desde el Plan Joven, por su ayuda e implicación en un tema tan importante en el desarrollo de políticas de juventud. estudio mediante fichas sirvió al grupo para avanzar, de un modo transversal, hacia un horizonte en el que la intervención, ya sea de carácter preventivo o asistencial, se realice de forma coherente y coordinada.

En este tiempo que nos ha tocado vivir, estamos asistiendo a una nueva problemática de la salud pública generada por la propia crisis, que pone en evidencia alguno de los factores de desventaja, como la situación socioeconómica de muchas familias, la derivada de los recortes de las distintas administraciones o la situación de la formación ocupacional, con las consecuencias que conlleva en las personas jóvenes en desventaja.

Como fruto final de la labor realizada, se redactó una serie de recomendaciones técnicas para orientar el trabajo futuro de los diferentes servicios y departamentos en la definición de programas, acciones o planes que contemplen actuaciones en los diferentes ámbitos incluidos en el informe.

\section{Metodología de trabajo}

La metodología utilizada en la elaboración del informe se basó en la coordinación interdepartamental e interinstitucional. De modo sintético, éstos fueron los pasos seguidos:

- Búsqueda de consenso en la definición conceptual.

- Búsqueda de datos relevantes en el análisis de la realidad estudiada.

- Análisis de los datos.

- Plasmación de los datos en informes, clasificados por ámbitos.

- Concreción de los factores o situaciones de desventaja y conductas de riesgo en fichas que facilitaran el estudio de la información. Cada ficha se estructuraba de la siguiente manera: a) descripción de la situación de desventaja o conducta de riesgo; b) datos; c) análisis de los datos; d) propuestas de intervención.

- Contraste del análisis y de las fichas con otros agentes, tanto municipales como de otras instituciones.

- Elaboración del documento final, y divulgación al personal técnico y a la representación política.

Los criterios metodológicos se han adecuado a la realidad encontrada, tratando siempre de reflejarla desde varios ámbitos, ya que la realidad juvenil no es algo que se pueda compartimentar, sino que debe analizarse desde una perspectiva integral, que nos permitirá, en última instancia, ofrecer una respuesta adecuada.

El informe no agotaba las situaciones de desventaja ni las conductas de riesgo, ya que algunas de ellas no permiten fácilmente su separación y diferenciación de las otras. 


\section{Documento de partida}

\subsection{Desventaja social y conductas de riesgo en las personas jóvenes}

Como se ha mencionado, una de las líneas de trabajo del II Plan Joven Municipal (en concreto, la no 4 , titulada 'Integración y socialidad') contemplaba, entre sus acciones, la configuración de un grupo de trabajo transversal sobre prevención y conductas de riesgo. Este equipo estaba conformado por personal técnico del Servicio de Policía Local (Departamento de Seguridad Ciudadana), el Servicio de Salud Ambiental (Departamento de Medio Ambiente y Espacio Público), el Departamento de Asuntos Sociales y de las Personas Mayores, la Unidad de Juventud (Servicio de Planificación Cultural y Fiestas) y el Departamento de Servicios a la Ciudadanía y Deportes (Servicio de Centros Cívicos y Servicio de Educación). Los objetivos de este grupo fueron tres:

- Elaborar un documento que facilitara la aproximación a los conceptos de desventaja social y de conducta de riesgo.

- Realizar un diagnóstico de la situación en Vitoria-Gasteiz.

- Estructurar un plan de acción en torno a la desventaja y las conductas de riesgo entre las personas jóvenes de la ciudad.

En respuesta al primero de esos objetivos, se redactó lo que denominamos documento de partida, fundamentado en dos trabajos que están considerados como referencias destacadas en la aproximación conceptual que nos ocupa: Jóvenes en situación de desventaja social: políticas de transición entre la construcción social y las necesidades de una juventud vulnerable, de René Bendit y Dermot Stokes (2004), y Las constelaciones de desventaja se hacen visibles en España, de Germán Gil Rodríguez (2007). No se trataba tanto de asumir ideológicamente lo que proponen, ni tampoco de trasladar su interpretación a la realidad de nuestra ciudad, como de que nos sirvieran para clarificar nuestro mapa conceptual y poder establecer un plan de intervención basado en una idea compartida.

\subsection{Juventud desfavorecida y concepto de desventaja social}

La definición de juventud desfavorecida está supeditada a la de desventaja, ya que ambos términos se hayan íntimamente relacionados: podemos identificar la juventud desfavorecida con aquella que vive en situaciones de desventaja. Entendemos la desventaja social como aquellos factores que forman parte de un proceso que empieza en la situación de normalidad; factores de desventaja que, si se acumulan, dan lugar a situaciones de vulnerabilidad y pueden acabar (en muchos casos, asociados a conductas de riesgo) en la exclusión social. La acumulación de factores en una persona y la asociación con conduc- tas de riesgo son los contextos que ayudan a explicar algunas situaciones.

El concepto de desventaja social está en el origen del proceso de exclusión social, del mismo modo que el de ventaja lo está en el de inclusión social. Implica el reconocimiento de que, a pesar del paso a la ciudadanía universal y a la provisión de bienestar, diversos grupos poblacionales pueden experimentar un acceso desigual a recursos tales como el empleo o las prestaciones. Esta situación genera vulnerabilidad, la cual ejerce una clara influencia sobre las oportunidades objetivas de la vida de las personas y, en consecuencia, explica la aparición de determinadas conductas de riesgo.

\subsection{La intervención ante la desventaja social}

Hoy día es evidente que tanto la educación -estimada como el instrumento más valioso para que la sociedad supere las diferencias sociales- como el trabajo -considerado el factor más importante para la integración social de las personas jóvenes- no han cumplido con las expectativas que podían generar como variables de superación de las condiciones de desventaja. La realidad ha venido a demostrar que la situación de desventaja social, especialmente la que afecta a las personas jóvenes, responde a una pluralidad de causas que hacen que la escuela/educación no pueda, por sí sola, solucionar las causas de la desigualdad, ni la desigualdad en sí misma. En el sistema educativo se concentran todas las modalidades de desventaja social, puesto que la educación se ve sometida a las múltiples exigencias, demandas y contradicciones de los elementos que en ella intervienen, aunque el sistema educativo no tiene los recursos ni la posibilidad de solucionar total o parcialmente las desventajas, ya que ello requiere políticas más integrales promovidas desde las instituciones.

Éstas y otras consideraciones nos llevan a la necesidad de prevenir de forma integral la aparición de situaciones de desventaja y, en consecuencia, a minimizar también la acumulación de conductas de riesgo. En este sentido, planteamos algunas recomendaciones:

- Considerar prioritarias la prevención y la intervención temprana. El desarrollo de los factores protectores que promueven la inclusión debe hacerse desde la primera infancia, momento en el que empiezan a acumularse las ventajas o desventajas del desarrollo humano.

- Los objetivos, enfoques y metodologías de los distintos servicios intervinientes -y en particular, del equipo de proximidad- deben ser transparentes y consensuados.

- Es necesario pensar en nuevos escenarios de provisión de los servicios, que partan del lugar en el que se encuentra la persona joven y la sitúen en el centro de la acción. Se producen en el contexto 
del individuo y en el ámbito donde convive. Por lo tanto, su recuperación depende de la propia persona, de la realidad donde vive, de su entorno y de sus relaciones.

- Hay que integrar servicios, sistemas y puntos de vista. Debe contarse con enfoques multidimensionales de carácter comunitario, que potencien el desarrollo de proyectos conjuntos y que, a su vez, faciliten el establecimiento de vínculos entre los mundos de la educación, el trabajo social, la formación y el empleo.

- Es necesaria una investigación adecuada y el diseño de mecanismos de evaluación (diagnóstico consensuado de la realidad de factores de desventaja y de las conductas de riesgo en las personas jóvenes en Vitoria-Gasteiz).

- Debe disponerse de los recursos apropiados para tener éxito en el trabajo con las personas más desfavorecidas.

- Las personas con responsabilidad técnica y política deben adoptar una perspectiva a largo plazo.

\section{Diagnóstico de la situación en Vitoria- Gasteiz y propuestas de intervención}

Las situaciones de desventaja y conductas de riesgo identificadas entre la población juvenil de VitoriaGasteiz se resumen en el Cuadro 1.

\section{1. Ámbito educativo}

\subsubsection{Conducta de riesgo: absentismo escolar y desescolarización}

En 2011, se dieron 492 casos de absentismo en Vitoria-Gasteiz, el 80,78\% del total del territorio alavés. De ellos, 409 (83,1\%) se localizaron en la red pública y $83(16,9 \%)$ en la concertada; el absentismo era mayor en la red pública que en la red concertada en todas las etapas de la educación básica. Por otro lado, los 14 casos de desescolarización detectados en el territorio alavés se dieron en centros escolares de la red pública de Vitoria-Gasteiz.

A este respecto, se plantean varias propuestas de intervención:

- Establecer un protocolo de actuación interinstitucional.

- Revisar la coordinación de los distintos servicios municipales involucrados.

- Primar las medidas preventivas frente a las reactivas.

- Informar a las familias.

- Desarrollar programas de sensibilización dirigidos a las familias desfavorecidas.

- Impulsar la participación de las familias.

- Impulsar los programas educativos que puedan influir en el éxito escolar.

- Impulsar las ayudas a las asociaciones de madres y padres de alumnado.

- Llevar a cabo tareas de mediación entre la institución escolar y la familia.

- Diseñar y aplicar planes de intervención psicosocial.

- Evaluar la situación de la desescolarización y del absentismo escolar, así como las intervenciones que se pongan en marcha en este terreno.

\subsubsection{Factor de desventaja: índice socioeconómico y cultural}

Una de las variables externas que influyen en el ámbito educativo es el índice sobre la condición

\begin{tabular}{|l|l|}
\hline \multicolumn{2}{|l|}{ Cuadro 1. Distribución de las situaciones de desventaja y conductas de riesgo por ámbitos } \\
\hline Ámbito & Situaciones de desventaja y conductas de riesgo \\
\hline Educativo & $\begin{array}{l}\text { - Absentismo escolar y desescolarización. } \\
\text { - Índice socioeconómico y cultural. }\end{array}$ \\
\hline - Dieta, hábitos alimentarios y obesidad. \\
- Conductas sexuales de riesgo. \\
- Consumo de drogas. \\
- Ausencia de ejercicio físico. \\
- Enfermedades no transmisibles. \\
- Ludopatía. \\
- Autovaloración de la salud negativa. \\
- Problemas de salud mental. \\
- Suicidio.
\end{tabular}

Fuente: Grupo de Trabajo Interdepartamental (2012). 
socioeconómica y cultural del alumnado (ISEC). Desde esta perspectiva, en la evaluación diagnóstica realizada en 2011 en Vitoria-Gasteiz, se pueden destacar los siguientes datos, correspondientes al alumnado de $2^{\circ}$ de ESO:

- A mayor ISEC, mejores resultados. Tal relación se mantiene en las cuatro competencias evaluadas: comunicación lingüística en euskara, comunicación lingüística en castellano, matemáticas y comunicación lingüística en inglés.

- La mayoría de los centros educativos se encuentran en torno al resultado esperado en relación con su ISEC.

- Hay centros que, aunque tienen el mismo nivel de ISEC, obtienen resultados académicos muy diferentes.

En torno al ISEC, se sugieren las siguientes propuestas de intervención:

- Tomar conciencia de que los centros educativos pueden lograr resultados más allá de lo esperado de acuerdo al ISEC del alumnado que escolariza.

- Elaborar intervenciones que redunden en la acción y la organización educativas de los centros.

- Definir la coordinación necesaria entre los distintos servicios municipales.

- Revisar la intervención educativa de los distintos servicios municipales.

- Desarrollar programas de sensibilización y formación para las familias.

- Priorizar las demandas de centros escolares con un ISEC medio-bajo o bajo.

- Fomentar la participación de las familias.

- Impulsar los programas educativos que puedan influir en el éxito escolar.

- Potenciar las ayudas a las asociaciones de madres y padres de alumnado para el desarrollo de actividades extraescolares y complementarias.

- Modificar la relación del alumnado con su entorno y favorecer la ocupación del tiempo de ocio en actividades educativas organizadas por los centros e instituciones del entorno.

\section{2. Ámbito de la salud}

\subsubsection{Factor de desventaja: dieta, hábitos alimentarios y obesidad}

Desde el punto de vista de salud pública, la principal preocupación por el estado nutricional es el exceso o inadecuación de la alimentación. La obesidad está asociada con las principales enfermedades crónicas de nuestro tiempo. A mayor obesidad, las tasas de morbilidad y mortalidad por esas enfermedades son mayores.
En materia de alimentación, se sugieren siete intervenciones:

- Fomentar los programas de educación para la salud en el medio escolar y en la comunidad.

- Cumplir el Programa del Niño Sano de o a 14 años.

- Realizar una información oral y escrita sobre hábitos saludables de alimentación.

- Incorporar contenidos sobre hábitos saludables de alimentación en las sesiones introductorias a los exámenes de salud programados por el Servicio de Salud escolar.

- Poner en marcha un programa de actividades preventivas con todas las personas mayores de 16 años que incluya la medición del índice de masa corporal (IMC).

- Ofertar a todas las personas con un IMC superior a 30 un control de la variación interanual (consulta, dietas, seguimiento).

- Mantener la colaboración entre el Ayuntamiento de Vitoria-Gasteiz y los centros de salud para impulsar la actividad física.

\subsubsection{Conducta de riesgo: conductas sexuales de riesgo}

Dos de las consecuencias negativas de la actividad sexual más relevantes desde el punto de vista de la salud son la transmisión de enfermedades sexuales y los embarazos no deseados. En 2009, en la Comunidad Autónoma del País Vasco se detectaron 95 infecciones gonocócicas y 112 casos de sífilis. En Álava, se da una tendencia creciente, especialmente, en el caso de la sífilis.

En este ámbito se proponen estas intervenciones:

- Mejorar la salud sexual y reproductiva mediante una educación sexual desde la etapa escolar.

- Cumplir el Programa de Actividades Preventivas.

- Mantener la asistencia, la educación y la prevención sobre conductas sexuales en los centros de atención a la mujer.

- Proporcionar, al menos una vez, información y consejo sobre métodos anticonceptivos.

- Mantener la oferta de consulta confidencial del VIH, tanto para la prueba como para el consejo.

- Facilitar información de las farmacias autorizadas para la realización del test rápido del HIV.

- Mantener la Asexoría de sexualidad.

- Mantener el trabajo de asesoramiento e información con el conjunto de mediadores/as y educadores/as que trabajan con jóvenes.

\subsubsection{Conducta de riesgo: consumo de drogas}

El consumo de drogas acarrea una gran cantidad de problemas a las personas que lo practican, lo que 
repercute no sólo en la esfera personal, sino también en el ámbito familiar, social, sanitario o de orden público. Cuando se habla de drogas, se suele prestar más atención a las drogas ilegales, pero no puede obviarse que muchos estudios advierten que sustancias como el alcohol y el tabaco son las que producen mayor mortalidad, enfermedades y discapacidades.

Las recomendaciones de intervención en el terreno de las drogodependencias son las siguientes:

- Implementar programas educativos relacionados con las drogas legales e ilegales.

- Promover acciones de sensibilización, información y formación para padres y madres.

- Controlar la publicidad y de la promoción indebida de bebidas alcohólicas y tabaco.

- Controlar el suministro y venta de bebidas alcohólicas a menores.

- Cubrir las necesidades de apoyo de los centros, para realizar proyectos e intervenciones preventivas.

- Incorporar la prevención del uso de drogas en el currículo de prevención de riesgos laborales en Formación Profesional.

- Garantizar apoyo y orientación a los equipos docentes

- Promover actividades con familias vulnerables y recursos personalizados de información y orientación psicológica y socioeducativa para familias

- Desarrollar proyectos de información, prevención y reducción de riesgos del consumo abusivo de alcohol y otras drogas en espacios festivos y de ocio.

- Impulsar la labor preventiva en las lonjas33.

- Fomentar los proyectos de educación de calle.

- Informar sobre consumo responsable y la oferta de programas de deshabituación.

- Mantener los contenidos sobre el consumo de alcohol y tabaco en las sesiones introductorias a los exámenes de salud programados por el Servicio de Salud escolar.

- Realizar y fomentar iniciativas sobre una vida sin tabaco, tanto en los centros escolares como en la comunidad en general.

- Promover los programas de deshabituación tabáquica individual y grupal.

- Llevar a cabo intervenciones educativas de cara a desarrollar la capacidad crítica de las personas jóvenes con respecto a la publicidad.

- Desarrollar iniciativas desde la edad escolar que fomenten la autoestima y la capacidad de afrontar los posibles problemas de la vida cotidiana.

\subsubsection{Factor de desventaja: ausencia de ejercicio físico}

La actividad y el ejercicio físico son considerados como una de las bases fundamentales para el buen estado de salud. La actividad física adecuada produce sentimientos positivos. Algunas formas de actividad física implican contacto interpersonal y favorecen el desarrollo de la dimensión social. En 2011, el 78,5\% de los chicos y chicas de entre 13 y 18 años de Vitoria-Gasteiz practicaban algún tipo de actividad físico-deportiva. Pero a estas edades ya había diferencias importantes de género en la distribución de las prácticas: los chicos eran más activos, siendo especialmente llamativa la bajada de actividad que se daba entre las chicas de más de 15 años.

Para remediar el déficit de ejercicio físico, se plantean las siguientes intervenciones:

- Poner en marcha talleres de educación para la salud en el ámbito escolar y en la comunidad, sobre la importancia que la actividad física tiene en la salud.

- Abordar de un modo transversal las intervenciones relacionadas con el estilo de vida saludable.

- Mantener la colaboración entre los centros de salud y el Ayuntamiento de Vitoria-Gasteiz ${ }^{4}$.

\subsubsection{Factor de desventaja: enfermedades no transmisibles}

De entre las diversas enfermedades graves no transmisibles -como el cáncer, las enfermedades cardiovasculares, las enfermedades crónicas o degenerativas y la diabetes mellitus tipo 1-, entre la población joven fundamentalmente se da la diabetes mellitus tipo 1. Esta hiperglucemia crónica se asocia a largo plazo con alteraciones en los ojos, los riñones, el sistema nervioso o el sistema circulatorio.

En este ámbito se recomienda:

- Fomentar iniciativas de prevención de enfermedades crónicas en la comunidad y talleres de promoción de la salud desde la etapa escolar.

- Ofrecer información actualizada a niñas, niños, adolescentes y personas adultas con diabetes mellitus tipo 1. Realizar una educación diabetológica que incluya el consejo dietético.

- Implementar el programa de prevención de la diabetes tipo 2, D-Plan.

- Dirigir el trabajo a la prevención de la diabetes tipo 2 t y de otras enfermedades crónicas no transmisibles. 


\subsubsection{Conducta de riesgo: ludopatía}

La adicción al juego se considera una dependencia de tipo emocional y pone en riesgo las áreas más importantes de la vida de una persona: trabajo, estudios, relaciones sociales, familia, pareja, economía, salud física y mental. En 2011, la edad media de las personas que acudieron a los servicios que atienden estas adicciones fue de 35 años. Por franjas de edad, un $14,75 \%$ de quienes acudieron tenían menos de 25 años, y alrededor de un $30 \%$, entre 25 y 35 años.

Cabe señalar también la presencia del juego en línea y los problemas que genera, sobre todo, entre la población joven (apuestas y juegos de rol).

En materia de ludopatía, éstas son las propuestas de intervención que se sugieren:

- Trabajar con la población general las conductas adictivas en relación con las máquinas tragaperras

- Prestar atención a la evolución del novedoso juego en línea.

- Realizar campañas dirigidas a la población afectada.

- Dar a conocer páginas web informativas a través de las que poder realizar consultas anónimas.

- Facilitar material y formación al personal sanitario y de los diferentes servicios sociales.

- Informar a la ciudadanía sobre la posible relación entre el consumo de sustancias y el consumo de juegos de azar.

- Realizar un estudio que permita conocer la relación entre juegos de azar y consumo de sustancias en la población general, y el porcentaje de población que se vería afectada por este tipo de 'multiconsumo'.

- Elaborar un estudio para conocer la incidencia de la ludopatía en la población joven y en la población escolar.

- Llevar a cabo campañas de prevención dirigidas a jóvenes y poner en marcha programas preventivos en el ámbito escolar.

\subsubsection{Factor de desventaja: autovaloración de la salud negativa}

La autovaloración de la salud refleja, de manera global, la apreciación que las personas tienen de su propia salud, incluyendo componentes subjetivos y objetivos. En 2011, uno de cada cuatro varones jóvenes manifestó que su salud era excelente, lo que representa una proporción dos veces mayor que entre las mujeres jóvenes.

A este respecto, se propone realizar intervenciones de educación para la salud, tanto en centros escolares como en la comunidad.

\subsubsection{Factor de desventaja: problemas de salud mental}

Los problemas de salud mental se asocian, en ocasiones, con conductas de riesgo como el consumo de sustancias tóxicas (toxicomanías) o el consumo de alcohol (alcoholismo). No son factores que aparezcan aislados, salvo en algunos casos excepcionales, sino que están ligados entre sí por relaciones causa-efecto, a veces de difícil discriminación. Las personas de 14 a 30 años atendidas en 2001 en dispositivos de psiquiatría del municipio de Vitoria-Gasteiz fueron 1.460. De ellas, 776 eran hombres y 684 mujeres jóvenes.

El tratamiento en los centros de alcoholismo o toxicomanías es un fenómeno casi exclusivamente masculino. En el resto de los centros de salud predominan las mujeres jóvenes.

Las propuestas de intervención en el área de salud mental son:

- Coordinar los servicios sociales de base municipales con los servicios de salud mental de Osakidetza.

- Trabajar la prevención desde edades tempranas.

- Promocionar la salud desde la etapa escolar.

- Disponer de un conocimiento de la evolución de la realidad.

- Mantener la Asesoría Psicológica para Personas Jóvenes como recurso preventivo de asesoramiento e información.

\subsubsection{Conducta de riesgo: suicidio}

La tasa de suicidios del territorio se ha incrementado en la CAPV, empujada principalmente por la situación de crisis actual. El suicidio está muy relacionado con la enfermedad mental. El riesgo de suicido es mayor en fases tempranas de la enfermedad y en los primeros seis meses, tras el alta de un ingreso. Las tasas de suicidio más elevadas se dan entre los 49 y 59 años, aunque está aumentando la incidencia en varones jóvenes entre los 15 y 24, y los 25 y 34 años. Además, en la población comprendida entre los 15 y los 44 años de edad, las autolesiones constituyen la cuarta causa de muerte y la sexta causa de mala salud y discapacidad.

Para hacer frente al riesgo de suicidio, se plantea realizar una labor preventiva.

\section{3. Ámbito judicial}

\subsubsection{Conducta de riesgo: accidentes}

La mayor frecuencia de las lesiones no intencionadas corresponde a las edades comprendidas entre los 15 y los 24 años. A partir de este tramo de edad, la horquilla tiende a disminuir. Los varones menores de 15 años se accidentan con más frecuencia en lugares públicos o de recreo. Las mujeres menores de 
14 años se accidentan en el colegio o en lugares de recreo, mientras que las que tienen entre 15 y 24 lo hacen en lugares públicos. Los accidentes de tráfico son la principal causa de muerte entre la población joven de ambos sexos con edades comprendidas entre los 15 y los 24 años.

Con el fin de atajar los accidentes, se sugiere:

- Realizar intervenciones educativas de corte preventivo en centros escolares y centros cívicos.

- En lo se refiere a la prevención de los accidentes de tráfico, se recomienda:

- Aumentar el uso de cascos de buena calidad.

- Incrementar la utilización de los cinturones de seguridad.

- Cumplir el Programa del Niño Sano de o a 14 años.

- Fomentar el uso de sistemas de seguridad y retención.

- Impulsar la educación vial.

- Llevar a cabo acciones de sensibilización entre jóvenes en el ámbito escolar y ciudadano para fomentar el comportamiento responsable como peatones, ciclistas y conductores/as.

- Realizar controles de alcoholemia preventivos.

- Poner en marcha un plan para ralentizar el tráfico en la ciudad.

\subsubsection{Conducta de riesgo: conductas 0 comportamientos antisociales que llevan a detenciones 0 imputaciones}

La desventaja, en este caso, viene dada por las conductas de riesgo que provocan la relación entre las personas jóvenes y la Policía. Esta realidad afecta tanto a personas jóvenes nacidas en el Estado español como a otras de origen extranjero, y en mucha mayor medida a los hombres jóvenes que a las mujeres. El 93,8\% de las personas jóvenes detenidas en 2011 fueron hombres y el 6,2\%, mujeres. En el caso de las imputaciones, el $87 \%$ fueron hombres y el $13 \%$, mujeres. Respecto a la nacionalidad, la mayoría eran personas autóctonas en el caso de imputaciones y de origen extranjero en el caso de las detenciones. Entre los motivos, destacan los delitos contra el patrimonio.

Las intervenciones propuestas en este ámbito son:

- Realizar un trabajo coordinado en red entre las distintas instituciones con competencias en la materia.

- Disponer de un conocimiento actualizado de la realidad.

- Realizar un trabajo preventivo de sensibilización.

- Fomentar el uso de medidas alternativas y en medio abierto entre jóvenes autores de infracciones y delitos.

\section{4. Ámbito sociolaboral}

5.4.1. Factor de desventaja: dificultad de acceso al mercado laboral por falta de cualificación profesional

El abandono de los estudios antes de obtener el título de Secundaria o Bachillerato conlleva una situación de desventaja en un mundo que cada vez exige una mayor capacitación. Según los datos del Gabinete de Estudios municipal, en torno al $30 \%$ de las personas jóvenes no tienen un título que las capacite para la entrada en el mercado de trabajo.

Las personas más jóvenes que abandonan el sistema escolar obligatorio son las más vulnerables a la hora de reintegrarse en el sistema formativo y, por tanto, las más proclives a carecer de una cualificación laboral. El incremento de las personas jóvenes inscritas en el paro y sin cualificación para el trabajo ha aumentado en los últimos años.

Es de resaltar la problemática que presentan algunas personas jóvenes de entre los 16 y los 20 años que, habiendo abandonado el sistema escolar o los cauces para la obtención de un título que les dé acceso al mercado de trabajo, no acaban de encontrar sitio ni en el mercado laboral, ni en centros de formación ocupacional, quedando totalmente desubicadas en una etapa fundamental de sus vidas.

Para atajar las dificultades de acceso al mercado laboral por déficits formativos, se sugiere:

- Tratar esta problemática desde una óptica preventiva, antes de que confluya con otros factores de desventaja y conductas de riesgo.

- Impulsar la coordinación con las y los responsables del mundo educativo y formativo.

- Proveer recursos a los centros que imparten programas de Cualificación Profesional Inicial (o sus equivalentes tras la aplicación de la Ley Orgánica para la Mejora de la Calidad Educativa).

- Mantener un conocimiento actualizado de la situación.

- Dotar de recursos de formación ocupacional a las estructuras municipales.

\subsubsection{Factor de desventaja: precariedad social}

La precariedad social a menudo está vinculada con la precariedad laboral y, en ocasiones, con las condiciones legales de determinados grupos sociales y el desconocimiento de los idiomas locales. En relación al grupo etario que nos interesa, la precariedad social puede entenderse como la presencia de factores sociales estructurales que colocan a las personas jóvenes en una situación de desventaja. Entre estos factores, se encuentran la escasez de recursos económicos y sociales, un bajo nivel educativo y los problemas de acceso a una vivienda o a otras necesidades básicas. En cuanto al nivel de estudios, en 2011 cabe destacar que un porcentaje importante 
de las personas jóvenes atendidas en los servicios municipales de Asuntos Sociales (40,9\%) no tiene estudios o tiene estudios inferiores al graduado escolar, y un $20,3 \%$ no sabe leer ni escribir.

Podríamos decir que el $9,4 \%$ de las personas jóvenes empadronadas en Vitoria-Gasteiz (3.523 personas) tienen problemas para cubrir sus necesidades básicas de alimentación, vestido, higiene y gastos personales. Estas personas son principalmente de nacionalidad extranjera ( $71,7 \%)$. Hay un colectivo de especial vulnerabilidad que se encontraría en una situación de pobreza absoluta: unas 250 personas jóvenes con una edad media de 21 años que no pueden cubrir sus necesidades básicas, ya que no tienen derecho a percibir la renta de garantía de ingresos.

En 2011, 339 jóvenes participaron en programas para la inserción sociolaboral, por presentar dificultades en esta área. Es relevante destacar que la gran mayoría (272, el 80,2 \%) participaron en programas prelaborales. Ese mismo año, 470 personas jóvenes fueron atendidas en la red de recursos de alojamiento existente, para apoyar a personas en situación de vulnerabilidad o riesgo de exclusión. De éstas, un $39,8 \%$ fueron atendidas en recursos de mínima exigencia (27 en Estrada y 160 en Aterpe), es decir, se encontraban en situación de exclusión social severa. En estos casos, confluyen especialmente las diversas situaciones de desventaja y conductas de riesgo. Por último, 11 jóvenes fueron atendidos en el piso semitutelado para jóvenes (de 18 a 23 años).

En materia de precariedad social, las propuestas de intervención del diagnóstico realizado en VitoriaGasteiz son:

- Mejorar la recogida de datos, a escala interdepartamental e interinstitucional, y cómo compartirlos.

- Incluir en la recogida de datos las variables de sexo, edad y nacionalidad.

- Crear un mapa de recursos para jóvenes en situaciones de desventaja en todos los ámbitos,

- Mejorar la difusión de la información acerca de los recursos existentes.

- Pasar de un ámbito prelaboral a uno de capacitación de habilidades profesionales.

- Impulsar las prácticas en empresas para jóvenes de origen extranjero.

- Posibilitar el acceso a viviendas rotacionales o de alquiler social, que permitan a las personas jóvenes mejorar su autonomía.

- Visibilizar las situaciones de desventaja de colectivos concretos.

- Promover programas de acompañamiento desde edades tempranas, que trabajen la prevención y empoderamiento a escala individual y comunitaria.
- Impulsar, con un enfoque preventivo, el trabajo comunitario coordinado entre los diferentes agentes que intervienen en cada situación.

- Trabajar desde los principios de autonomía y empoderamiento de la juventud: ofrecer un acompañamiento, no entendido como supervisión cercana, sino como apoyo para fomentar la autonomía ("que vuelen solos").

- Propiciar la participación de personas jóvenes en lo que se vaya a diseñar e implementar.

- Potenciar la formación en las familias.

- Reducir la brecha digital: invertir en alfabetización digital.

\subsubsection{Factor de desventaja: paro o precariedad laboral}

La situación en la que se encuentran las personas jóvenes frente al mundo laboral puede suponer una desventaja, que afecta a uno de los ejes principales de su proceso de emancipación. Al mismo tiempo, es de resaltar la precariedad laboral que se centra especialmente en las personas jóvenes: precariedad en cuanto a las condiciones laborales (contratos temporales, frecuentes salidas y entradas en el mercado de trabajo, peores puestos) y precariedad en cuanto a las condiciones salariales.

Si observamos los datos de la CAPV facilitados por Eustat (2012), en el tercer trimestre de 2012 el paro entre las personas jóvenes llegó al 36,4\%, y la actividad y la ocupación bajaron a límites que no se conocían desde los años noventa: un 15,5\% de ocupación y un $25 \%$ de actividad. La relación entre estos datos y los correspondientes a quienes abandonan los estudios formativos puede abocar a la exclusión social de algunas personas jóvenes.

Frente a los problemas descritos, se propone:

- Considerar de forma integral los problemas que afectan a las personas jóvenes en el ámbito formativo y laboral.

- Coordinar las actuaciones con las instituciones responsables de empleo.

- Realizar actividades preventivas en coordinación con las instituciones educativas.

- Mantener los programas de formación ocupacional.

\section{5. Ámbito sociofamiliar}

\subsubsection{Factor de desventaja: situación familiar problemática (adolescentes en situación o en riesgo de desprotección)}

Las situaciones familiares problemáticas se definen como factores de rigidez o dificultades en la socialización familiar que provocan una situación de des- 
ventaja en la persona adolescente y joven. Pueden ir acompañadas de otros factores de desventaja, como las dificultades psicológicas o la falta de habilidades.

Dentro de las situaciones familiares problemáticas, se encuentra la desprotección. En 2011 el Servicio de Infancia y Familia atendió a 220 personas, con edades comprendidas entre los 14 y los 29 años, pertenecientes a familias en las que existía una situación de desprotección infantil o de riesgo de desprotección. De éstas, 103 (un 46,8\%) eran menores de edad (de entre 14 y 17 años); y 114 (de entre 18 y 29 años), madres/padres jóvenes. Por su parte, en el Servicio de Intervención Socioeducativa (SISE) fueron atendidas 126 personas jóvenes pertenecientes a familias que precisaban apoyos por carencias y dificultades en habilidades parentales o sociales. El $36 \%$ de ellas formaban hogares monoparentales. Por último, 388 adolescentes de entre 14 y 19 años fueron atendidos en el Programa de Educación de Calle.

Las propuestas de intervención ante este factor de desventaja pasan por:

- Mejorar la coordinación entre la administración municipal y otras instituciones públicas.

- Diseñar y promover medidas de apoyo a las familias jóvenes en la crianza y educación de sus hijos e hijas.

- Fomentar la detección temprana de situaciones de posible desprotección infantil y adolescente.

- Llevar a cabo campañas de sensibilización dirigidas a prevenir el maltrato infantil y adolescente.

- Incrementar la participación activa y directa de las personas menores de edad y jóvenes en la vida social de la ciudad.

- Ofrecer apoyo individualizado de personas referenciales a las y los jóvenes que, al cumplir la mayoría de edad, dejan de estar bajo la protección de los recursos y programas para menores.

- Desarrollar un acompañamiento flexible, facilitando la adquisición de autonomía por parte de las personas jóvenes.

\subsubsection{Factor de desventaja: discriminación por sexo, estereotipos de género y violencia de género}

La discriminación por sexo puede producirse junto con otras situaciones de desventaja, como las dificultades de tipo psicológico. En el caso de mujeres de origen extranjero, además, se pueden dar dificultades añadidas, asociadas a los requisitos legales para residir o trabajar, factores culturales y desconocimiento de los idiomas locales.

De los casos nuevos atendidos en 2011 en los Servicios Sociales Municipales por violencia de género, un 45,6\% eran mujeres jóvenes (14-29 años), la mayoría de las cuales (un 67,4\%) eran extranjeras, por lo que presentaban una mayor vulnerabilidad. Un 29,2\% de las mujeres víctimas de violencia de género que precisaron acogida inmediata eran mujeres jóvenes, menores de 29 años. Dos de las nueve mujeres que ingresaron en un piso de emergencia social específico para víctimas de violencia de género también eran jóvenes; ambas eran extranjeras, tenían un/a hijo/a menor a su cargo y escasos recursos económicos. Más de la mitad de las mujeres a las que se les adjudicó un terminal GPS eran jóvenes.

Un $25 \%$ de las mujeres atendidas en el Servicio de Atención Psicológica por ser víctimas de maltrato por parte de su pareja/novio eran jóvenes. La edad media de las mujeres víctimas de agresiones sexuales atendidas en el Servicio de Atención Psicológica fue de 27 años. Un $18 \%$ de los hombres que agredieron a sus parejas y fueron atendidos en el Servicio de Atención Psicológica eran menores de 30 años.

En materia de discriminación sexual y violencia de género, se sugieren las siguientes pautas de intervención:

- Reforzar las intervenciones preventivas con adolescentes y jóvenes.

- Crear foros de reflexión compartidos entre los diversos ámbitos y agentes implicados.

- Desarrollar intervenciones coordinadas entre los diversos ámbitos (social, educativo).

- Realizar campañas de sensibilización.

- Trabajar con la privacidad en los nuevos usos de las nuevas tecnologías (redes sociales).

- Dotar de más recursos de apoyo para la prevención en violencia de género.

- Poner en marcha intervenciones dirigidas a paliar la mayor vulnerabilidad de las mujeres extranjeras víctimas de violencia de género.

\subsubsection{Factor de desventaja: dificultades por discapacidad y dependencia}

La desventaja ligada a la discapacidad y la dependencia presenta una casuística muy variada y nos habla de situaciones difícilmente agrupables. En 2011, en Vitoria-Gasteiz había 706 personas jóvenes con un grado de discapacidad reconocido, de las cuales 430 eran hombres y 276 mujeres. En total, 450 tenían un grado de discapacidad de entre el $33 \%$ y el $64 \%$; y 256 , un grado por encima del $65 \%$. Por tramos de edad, 153 eran jóvenes de entre 14 y 18 años; 259 tenían entre 19 y 24 años; y 294, entre 15 y 29 años. Las principales causas de discapacidad y dependencia estaban asociadas a alteraciones o enfermedades físicas (537 personas jóvenes) y discapacidades intelectuales (158).

Para abordar las dificultades asociadas a la discapacidad y la dependencia juveniles, cabe sugerir la mejora de la coordinación entre las asociaciones que trabajan en el ámbito de la salud y las del ámbito de la discapacidad, y entre las distintas instituciones con competencias en este ámbito. 


\section{Bibliografía citada}

BENDIT, R.; y STOKES, D. (2004): “Jóvenes en situación de desventaja social: políticas de transición entre la construcción social y las necesidades de una juventud vulnerable", Revista de Estudios de Juventud, $\mathrm{n}$ 0 65, págs. 115-131 [<http://www.mtas.es/ injuve/biblio/revistas/Pdfs/numero65/ tema7.pdf $\rangle]$.

EUSTAT (2012): Estadística Municipal de Población Activa ([«http://www.eustat.es/estadisticas/ tema_131/opt_o/ti_Actividad_ocupacion_y_ paro/temas.html>]).

GIL RODRÍGUEZ, G. (2007): "Las constelaciones de desventaja se hacen visibles en España”, Revista de Estudios de Juventud, nㅡ 77, págs. 103-121 [<http://www.injuve.mtas. es/injuve/contenidos.downloadatt. action?id=161890736〉].
GRUPO DE TRABAJO INTERDEPARTAMENTAL (2012): Jóvenes en la encrucijada de la desventaja: análisis de las situaciones de desventaja social y conductas de riesgo en Vitoria-Gasteiz, Vitoria-Gasteiz, Ayuntamiento de VitoriaGasteiz [<http://www.vitoria-gasteiz.org/ wbo21/http/contenidosEstaticos/adjuntos/ es/88/10/48810.pdf >].

SERVICIO DE JUVENTUD (2010): II Plan Joven Municipal de Vitoria-Gasteiz 2010-2012, Vitoria-Gasteiz, Ayuntamiento de Vitoria-Gasteiz.

UNIDAD DE JUVENTUD (2013): Gasteizko Udaleren III. Gazte Plana 2013-2015 / III Plan Joven Municipal de Vitoria-Gasteiz 2013-2015, Vitoria-Gasteiz, Ayuntamiento de VitoriaGasteiz [<http://www.vitoria-gasteiz.org/ wbo21/http/contenidosEstaticos/adjuntos/ es/36/34/53634.pdf >]. 